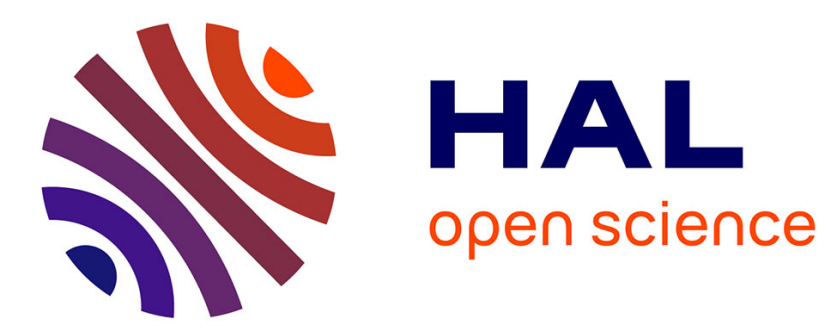

\title{
Équithérapie et autisme
}

L. Hameury, P. Delavous, Benoît Testé, C. Leroy, J.-C. Gaboriau

\section{To cite this version:}

L. Hameury, P. Delavous, Benoît Testé, C. Leroy, J.-C. Gaboriau. Équithérapie et autisme. Annales Médico-Psychologiques, Revue Psychiatrique, 2010, 168 (9), pp.655. 10.1016/j.amp.2009.12.019 . hal-00690278

\section{HAL Id: hal-00690278 \\ https://hal.science/hal-00690278}

Submitted on 23 Apr 2012

HAL is a multi-disciplinary open access archive for the deposit and dissemination of scientific research documents, whether they are published or not. The documents may come from teaching and research institutions in France or abroad, or from public or private research centers.
L'archive ouverte pluridisciplinaire HAL, est destinée au dépôt et à la diffusion de documents scientifiques de niveau recherche, publiés ou non, émanant des établissements d'enseignement et de recherche français ou étrangers, des laboratoires publics ou privés. 


\section{Accepted Manuscript}

Title: Équithérapie et autisme

Authors: L. Hameury, P. Delavous, B. Teste, C. Leroy, J.-C. Gaboriau

PII: $\quad$ S0003-4487(10)00254-4

DOI: $\quad$ doi:10.1016/j.amp.2009.12.019

Reference: AMEPSY 1223

To appear in: $\quad$ Annales Médico-Psychologiques

Received date: $\quad 26-8-2009$

Accepted date: $\quad$ 2-12-2009

Please cite this article as: Hameury L, Delavous P, Teste B, Leroy C, Gaboriau J-C, Équithérapie et autisme, Annales medio-psychologiques (2010), doi:10.1016/j.amp.2009.12.019

This is a PDF file of an unedited manuscript that has been accepted for publication. As a service to our customers we are providing this early version of the manuscript. The manuscript will undergo copyediting, typesetting, and review of the resulting proof before it is published in its final form. Please note that during the production process errors may be discovered which could affect the content, and all legal disclaimers that apply to the journal pertain. 
Figure 1

\section{EVALUATION EFC - score global et par enfant} 6 enfants - 19 séances

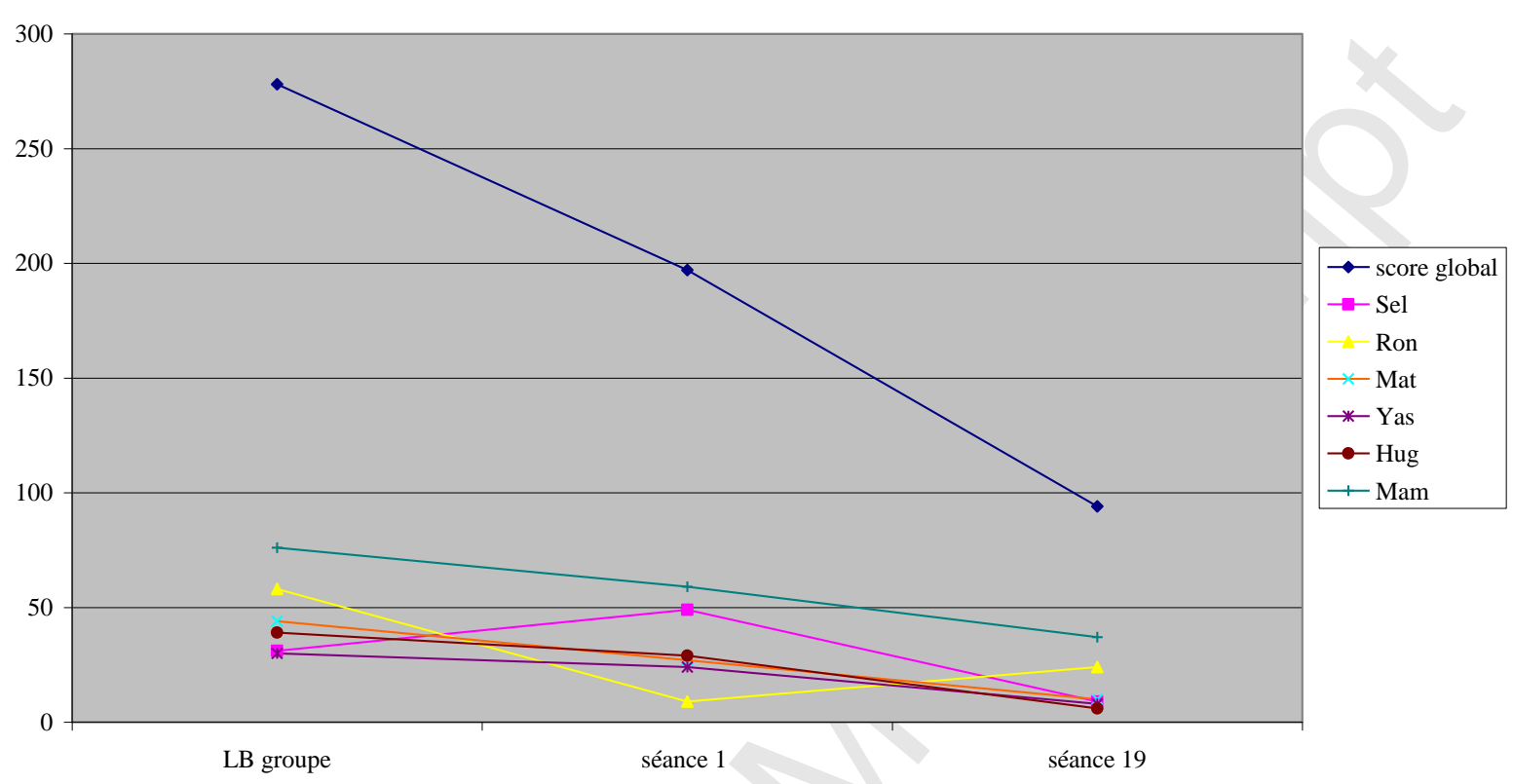


Figure 2

\section{EVALUATION EFC - score par fonction}

6 enfants - 19 séances

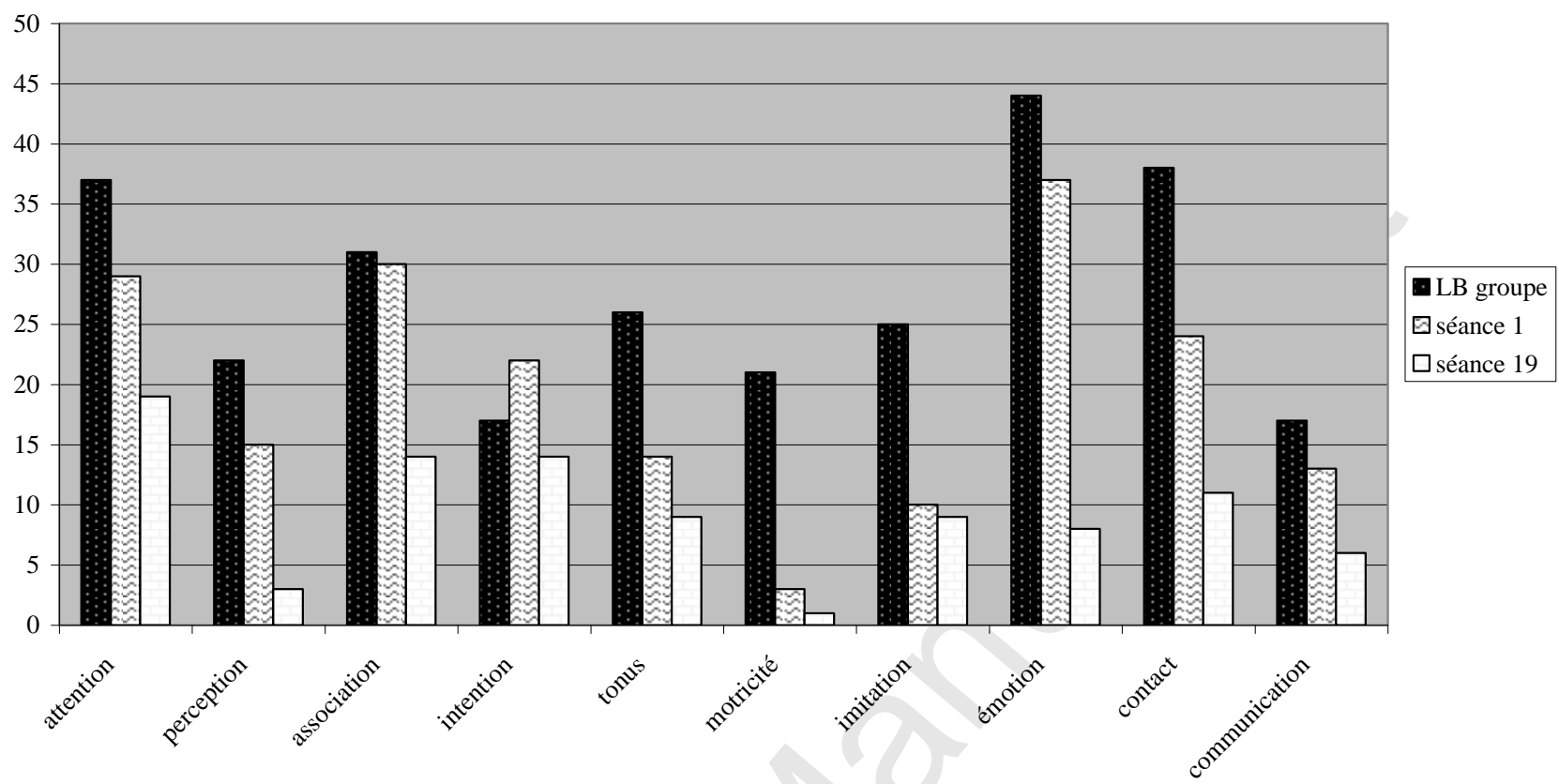


Figure 3

EVALUATION ECA groupe - score déficience relationnelle global et par enfant 6 enfants - 19 séances

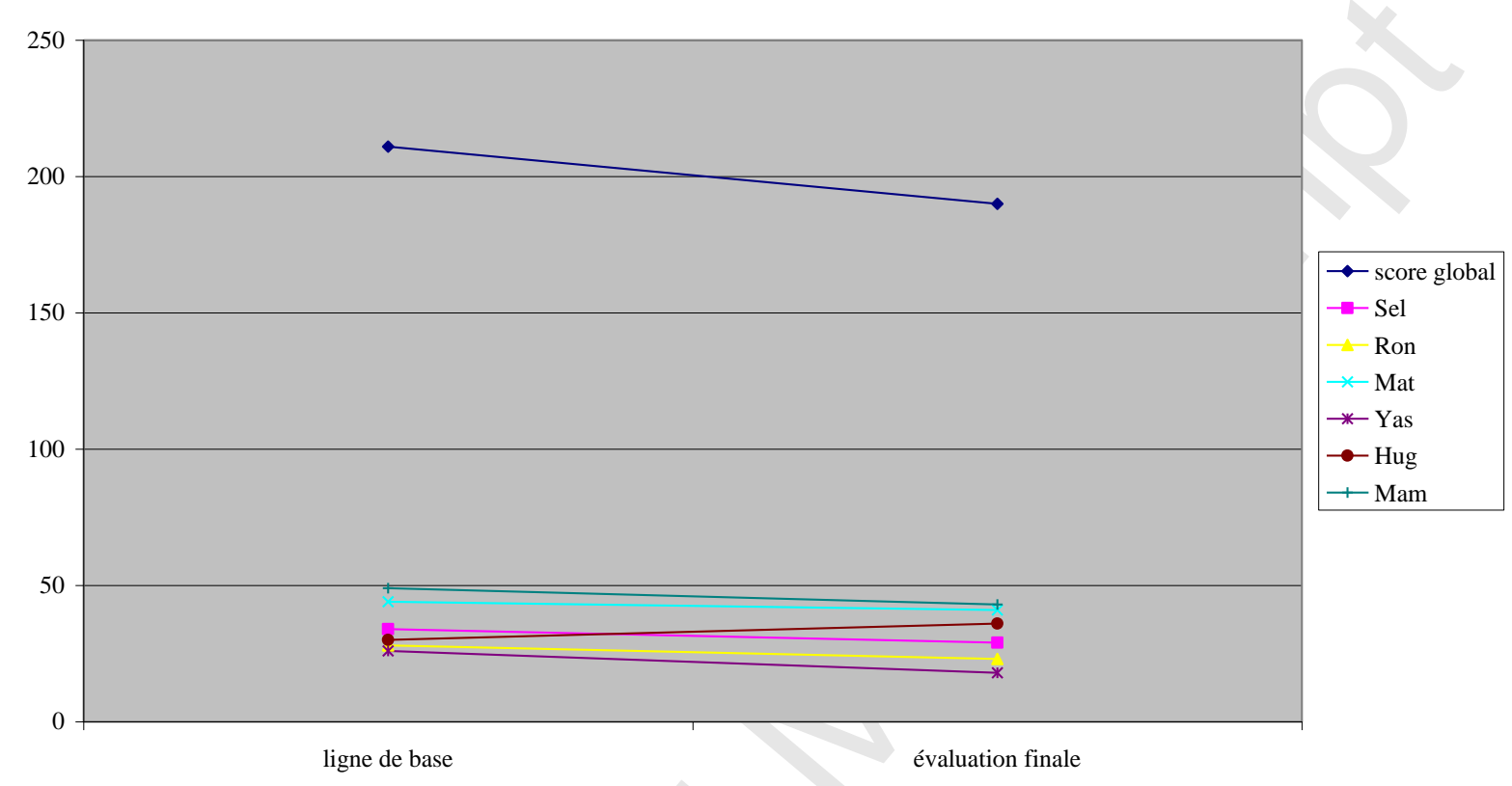


Figure 4

4 enfants - 30 séances

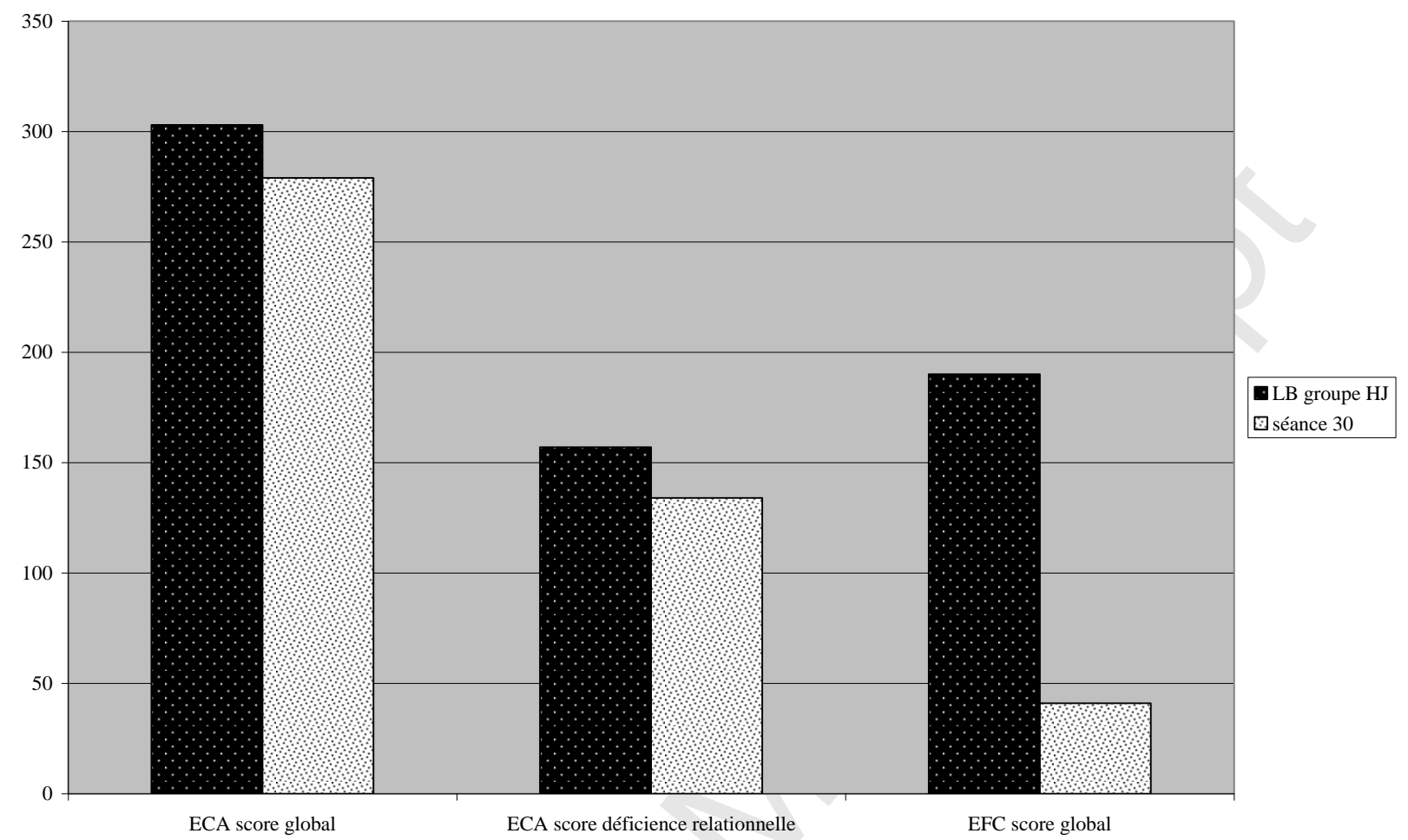




\section{Mémoire}

Équithérapie et autisme

Equine assisted therapy and autism

L. Hameury, P. Delavous, B. Teste, C. Leroy, J.-C. Gaboriau

Auteur correspondant: Centre universitaire de Pédopsychiatrie, CHRU Tours, 37044 Tours Cédex 9, France

tél. : 0247478847

fax : 0247478292

Adresse email : 1.hameury@chu-tours.fr

Texte reçu le 26 août 2009 ; accepté le 2 décembre 2009

Remerciements au Pr Lelord, à D. Bertho, C. Mahé, ainsi qu'aux poneys du poney-club La Galopade, 37300 Joué-les-Tours, France

\section{Résumé}

L'activité d'équithérapie est utilisée en complément des soins réalisés au centre de pédopsychiatrie. Elle est inclue dans le programme thérapeutique établi pour chaque enfant, dans le cadre d'un projet de soins personnalisé.

L'objectif est d'appliquer les principes et les techniques thérapeutiques développées pour les troubles du développement de l'enfant dans un cadre extrahospitalier, une ambiance apaisante et ludique, et avec l'aide du cheval comme médiateur.

Une évaluation quantitative des résultats a été réalisée pour six enfants présentant un trouble autistique. Les résultats montrent une nette amélioration dès la première séance d'équithérapie. Toutes les fonctions impliquées dans le développement sont améliorées, surtout celles concernant la communication, l'imitation, la régulation perceptive, émotionnelle et motrice.

L'équithérapie constitue donc une rééducation efficace de la communication et de la socialisation, ainsi que de la régulation cognitivo-émotionnelle. Elle permet des échanges appropriés, des acquisitions cognitives, sensorielles et motrices. L'enfant est stable, apaisé par 
les mouvements du poney, il sollicite l'équithérapeute dans une interaction adaptée et prend conscience de l'effet de ses actions sur le poney, excellent cothérapeute favorisant aussi l'attachement.

Mots clés : Autisme atypique ; Autisme infantile ; Équithérapie ; Spectre autistique ; Troubles envahissants du développement

\begin{abstract}
Equine assisted therapy is used beside therapies realised at the Child Psychiatry Center and included into individualised therapeutic program.

The goals are to adapt therapeutic principles outside hospital and with pony as mediator in order to exercise adjustment and control systems: Communication and relationships with others, cognitive regulation (attention, mental representation, understanding causality, anticipation), motor regulation (tonus, body consciousness, postural adjustment, balance, coordination), emotional regulation (self esteem, relaxation, pleasure, coping emotionally with problems, expressing and sharing emotions), adjusting to change.

Varied activities with tranquil and easy-going ponies were realised in a calm secure environment by three therapists with experience in horseback riding for a group of four children. An individual plan targeting specific goals was worked out for each child.

Results were evaluated with standardized tools (Behavior Function Inventory and Behavior Summarized Evaluation Scale) for six children with autistic spectrum treated at the day-care hospital which participated to equine assisted therapy.

Improvements are obtained since the first session and concerns all developmental functions, especially communication, imitation, perceptual, emotional and motor adjustment.

With his/her pony the child is stable, calms down, adjusts his/her interactions, is more aware of the effects of his/her actions, and optimal sensory integration can take place.

So, equine assisted therapy is an efficient remediation of the neurophysiologic functions involved in developing communication and social skills, and cognitivo-emotional regulation.
\end{abstract}

Keywords: Atypical autism; Autism; Autistic spectrum; Equine assisted therapy; Pervasive developmental disorders 


\section{Introduction}

L'équithérapie est une activité de soins reconnue par les structures publiques de santé, qui fait l'objet de travaux de recherche depuis une trentaine d'années. Elle s'inscrit dans un programme thérapeutique et son objectif est «d'agir sur le fonctionnement psychique du patient par des moyens psychiques et corporels » à l'aide de la médiation du cheval (Société française d'Équithérapie).

Elle est différente du loisir adapté, dont l'objectif n'est pas de soigner, ainsi que d'autres types d'interventions associant le cheval, et dont les orientations sont kinésithérapeutique (hippothérapie), psychomotrice (thérapie avec le cheval, TAC), ou sociale (rééducation par l'équitation, RPE).

Les vertus thérapeutiques du cheval sont reconnues depuis l'Antiquité et de nombreux auteurs ont décrit les bénéfices apportés par cet animal. C'est dans les années 1950 que s'est développée l'utilisation du cheval comme médiateur thérapeutique. En France, Renée de Lubersac, psychomotricienne, et Hubert Lallery, kinésithérapeute, ont fait connaître par leur travail cette nouvelle discipline thérapeutique [5,6]. Différentes études ont suivi et les applications en pédopsychiatrie se sont développées [7]. Le cadre et les limites thérapeutiques et déontologiques se sont précisées : «L'utilisation du cheval dans un dessein thérapeutique n'est pas l'apprentissage de l'équitation. » L'équithérapie est une «thérapie nécessitant une prescription médicale et du personnel paramédical spécialisé » [5]. L'équithérapeute doit être diplômé du domaine médico-social et avoir une connaissance et une expérience approfondies du cheval. La Société française d’Équithérapie a établi une charte d'éthique et de déontologie des équithérapeutes.

Les bénéfices constatés concernent les domaines neuromusculaire, relationnel, perceptif, attentionnel, émotionnel [10]. Le cheval est reconnu comme médiateur, source de sensations et d'apaisement, support d'identification et de socialisation, équivalent d'objet transitionnel, ayant un rôle de «holding », c'est-à-dire de portage, de bercement, de soutien, mais aussi de «handling », puisque en tant qu'objet relationnel, il favorise la création de liens, mais aussi l'indépendance, l'autonomisation, l'initiative. «Les activités reliées à l'animal minorent les atteintes physiques et psychiques liées au handicap » [4].

Sur ces bases théoriques, l'activité d'équithérapie a débuté au Centre universitaire de Pédopsychiatrie du CHRU de Tours en 1981, sous la direction du Pr Lelord, pour 13 enfants présentant des troubles graves du développement (autisme et troubles apparentés, retards avec 
troubles associés), avec des objectifs thérapeutiques portant sur la communication, l'attention, la confiance en soi, la motricité. Malgré les résultats positifs, cette expérience n'avait pu être prolongée pour des raisons matérielles. Elle a pu reprendre fin 2004, dans le cadre des soins à l'hôpital de jour.

\section{Objectifs et modalités}

Cette thérapie a été proposée à six enfants présentant des troubles envahissants du développement (autisme, autisme atypique, autres troubles envahissants du développement), et une évaluation quantitative des résultats a été réalisée à l'aide d'instruments déjà utilisés pour les travaux de recherche clinique dans le domaine des troubles du développement de l'enfant [9].

Il s'agissait d'appliquer les principes et les techniques thérapeutiques développées depuis de nombreuses années pour les troubles du développement de l'enfant [2,8] dans un cadre extrahospitalier, une ambiance apaisante et ludique, et avec l'aide du cheval comme médiateur afin d'exercer la communication et la relation avec autrui, l'adaptation à l'environnement, la régulation de l'activité et du comportement moteur, l'attention, la planification des actions, la conscience du corps (schéma corporel, organisation spatiale, coordination, équilibre, sensibilité proprioceptive, praxies fines, régulation du geste), la confiance en soi, la détente, la régulation émotionnelle, le partage des émotions, ainsi que les connaissances autour de l'animal. Ces objectifs sont en concordance avec ceux définis dans le projet thérapeutique individuel pour chaque enfant et sont appliqués également aux autres thérapies proposées à l'enfant.

L'activité d'équithérapie est utilisée en complément des soins réalisés au centre de pédopsychiatrie. Elle est inclue dans le programme thérapeutique établi pour chaque enfant, dans le cadre d'un projet de soins personnalisé défini en fonction des résultats des évaluations initiales puis des réévaluations du développement.

Elle est réalisée par trois thérapeutes du centre de pédopsychiatrie (un médecin pédopsychiatre, deux infirmiers spécialisés en pédopsychiatrie), formés aux thérapies d'échange et de communication (Thérapie d'Echange et de Développement, Barthélémy et al. 1995), et ayant une expérience en équitation.

Les parents sont associés au projet et peuvent suivre les progrès réalisés par leur enfant en visionnant les enregistrements vidéos réalisés lors de certaines séances (début, milieu et fin d'année scolaire). 
Le thérapeute doit être calme, attentionné, prévisible, étayant, et utiliser des stratégies psycho-éducatives adaptées. Il favorise l'échange, l'attention, la mise en œuvre des compétences de l'enfant, l'invite à s'exprimer par le langage et à partager ses émotions. Il l'encourage à réaliser l'activité proposée en s'ajustant à sa disponibilité et à ses réactions émotionnelles. Il tient compte de la fatigabilité de l'enfant et respecte une progressivité dans la difficulté, l'aide à surmonter ses peurs et à prendre confiance, fait varier en souplesse les activités et le cadre.

La sécurité est une préoccupation constante, nécessitant du thérapeute une grande vigilance, une anticipation des risques basée sur une bonne connaissance du cheval et de ses réactions : ne quitter des yeux ni l'enfant ni le poney, tenir le poney tant que l'enfant n'a pas la stabilité et l'autonomie requises ainsi que dans les espaces non clos.

Le poney doit être calme, tranquille, peu craintif, facile, gentil, tolérant, sérieux et attentif, de taille adaptée. Il est doux, chaud, agréable à toucher, attachant, réservé, mais réceptif à la relation. Il accepte facilement l'enfant, le porte, le balance, le berce, lui transmet des sensations. Il a aussi ses propres besoins et peut exprimer sa volonté et son désaccord. Il favorise le lien affectif et la prise de conscience d'autrui.

Les activités autour du poney sont très variées : observation des poneys dans leur box et en liberté, pansage (brossage, nettoyage), conduite à pied, monte en manège (espace fermé avec peu de distracteurs), et à l'extérieur en terrains variés (espaces ouverts et riches en stimulations), jeux divers en groupe (jeux d'adresse et de direction, jeux de poursuite, passage de petits obstacles, jeux d'échange de ballon, etc.). Les activités se font la plupart du temps à l'allure du pas, lente et rassurante; le trot et le galop, allures rapides, sautantes, riches en stimulations corporelles, ne sont utilisées que dans un but de stimulations motrices et vestibulaires, sur des temps très courts, avec de grandes précautions (poney toujours tenu par le thérapeute, surveillance étroite de l'équilibre et de la réaction émotionnelle de l'enfant). L'ambiance doit toujours être tranquille et sereine.

\section{Patients et méthode}

L'évaluation quantitative des résultats a concerné six enfants, âgés de cinq ans à sept ans, présentant un trouble envahissant du développement selon la CIM-10 (deux enfants avec autisme et retard léger, un enfant avec autisme et retard moyen, deux enfants avec autisme atypique, un enfant avec autre trouble envahissant du développement). Les scores d'autisme à la CARS (Childhood Autism Rating Scale) [11] variaient de 28 à 42. 
Ces six enfants recevaient des soins à l'hôpital de jour à temps partiel, cinq d'entre eux étaient scolarisés à temps partiel en école maternelle avec projet personnalisé de scolarisation et aide individualisée.

Les enfants ont participé aux séances d'équithérapie le mardi après-midi de 14 heures à 15 heures, une semaine sur deux, accompagnés de trois thérapeutes.

L'évaluation quantitative a été effectuée à l'aide d'instruments validés : l'échelle d'Évaluation des Comportements Autistiques (ECA) [3], et la grille d'Évaluation Fonctionnelle des Comportements (EFC) [1] :

- l'ECA comporte 29 items, cotés de un à cinq selon la fréquence du comportement autistique. Elle a été cotée par les soignants sur le groupe avant la première séance (ligne de base), et après la dernière (évaluation finale). Le score global et le score «déficience relationnelle » (items 1 à $6,8,9,12,23,24,26,28)$ ont été analysés ;

- grille de 36 items issus de la grille d'Évaluation Fonctionnelle des Comportements (EFC), cotés de zéro à quatre selon la fréquence du comportement autistique. Elle a été cotée par les soignants sur le groupe hôpital de jour avant la première séance (ligne de base), puis par les équithérapeutes d'après les enregistrements vidéos des séances d'équithérapie (première séance et $19^{\mathrm{e}}$ séance pour les six enfants, $30^{\mathrm{e}}$ séance pour quatre d'entre eux qui ont poursuivi une année supplémentaire). Le score global et les scores par fonction (attention, perception, association, intention, tonus, motricité, imitation, émotion, contact, communication) ont été analysés.

\section{Résultats}

Le score global à l'EFC est notablement amélioré pour l'ensemble des six enfants entre l'évaluation initiale sur le groupe hôpital de jour et la première séance d'équithérapie (29\%), ainsi qu'entre la première séance et la $19^{\mathrm{e}}$ séance (52\%). Une nette diminution entre le score en groupe et les scores en séances est observée pour tous les enfants (66 \% entre ligne de base et $19^{\mathrm{e}}$ séance). La figure 1 montre l'évolution des scores obtenus lors de la ligne de base sur le groupe hôpital de jour (LB groupe), lors de la séance 1 et lors de la séance 19 pour l'ensemble des six enfants et pour chaque enfant. Le score global est calculé en additionnant les notes des 36 items de la grille d'Évaluation Fonctionnelle des Comportements (EFC) et figure en ordonnées.

Le score global à la dernière séance est inférieur ou égal à dix pour quatre enfants. Seul un enfant a un score à la première séance plus élevé que le score de la ligne de base sur 
le groupe, du fait d'une importante réaction émotionnelle face au poney, rapidement surmontée lors des séances suivantes.

Selon les enfants, la diminution des scores entre ligne de base (LB) et dernière séance varie de $51 \%$ à $85 \%$ du score initial.

Figure 1

Pour toutes les fonctions, il y a une diminution nette des scores entre ligne de base sur le groupe (LB), première séance et $19^{\mathrm{e}}$ séance. La figure 2 montre l'amélioration des scores par fonction pour l'ensemble des six enfants. Les scores sont calculés pour chacun des 36 items de l'Évaluation Fonctionnelle des Comportements (EFC), puis regroupés par fonction. Les chiffres en ordonnées correspondent à la somme des notes des items pour chaque fonction. Les différentes fonctions figurent en abscisse. Les scores obtenus lors de la ligne de base sur le groupe hôpital de jour (LB groupe), lors de la séance 1 et lors de la séance 19 sont comparés.

La différence entre le score en groupe et le score en équithérapie dès la première séance est nette pour les fonctions attention (22\%), perception (32\%), tonus (46\%), motricité $(86 \%)$, imitation (60\%), émotion (16\%), contact (37\%), communication $(24 \%)$. Lors de la première séance, la fonction intention (30\%) est davantage perturbée et la fonction association n'est pratiquement pas améliorée (3\%).

Entre ligne de base et $19^{\mathrm{e}}$ séance, l'amélioration est importante pour toutes les fonctions (de $49 \%$ à $95 \%$ ). La fonction intention est la moins améliorée (18\%). Les fonctions les plus améliorées sont la perception (86\%), le tonus (65\%), la motricité (95\%), l'imitation (64\%), l'émotion (82\%), le contact (71\%), la communication (65\%).

Figure 2

L'évaluation des comportements autistiques sur le groupe hôpital de jour à l'aide de l'ECA met en évidence une légère diminution du score global pour l'ensemble des enfants ( $2 \%)$, et une diminution plus importante pour le score «déficience relationnelle » $(8 \%)$. La figure 3 illustre l'évolution du score déficience relationnelle obtenu lors de la ligne de base et à la fin des séances (évaluation finale) sur le groupe hôpital de jour pour l'ensemble des six enfants et pour chaque enfant. Le score «déficience relationnelle» (en ordonnées) est la somme des notes des 13 items de l'ECA évaluant la déficience relationnelle. 
Figure 3

Pour les quatre enfants ayant bénéficié de 30 séances, les scores entre ligne de base et cotation sur le groupe hôpital de jour après la $30^{\mathrm{e}}$ séance montrent une amélioration du score global ECA $(8 \%)$ et du score ECA « déficience relationnelle $(15 \%)$. Pour le score global EFC, la diminution des scores est très importante $(78 \%)$ entre ligne de base et $30^{\mathrm{e}}$ séance, et elle est de $34 \%$ entre les séances 19 et 30 . La figure 4 met en évidence l'amélioration obtenue après 30 séances. En ordonnées figurent les scores à l'Échelle d'Évaluation des Comportements Autistiques (ECA), score global (somme des notes des 29 items), et score «déficience relationnelle » (somme des notes des 13 items évaluant la déficience relationnelle), ainsi que le score global à l'Évaluation Fonctionnelle des Comportements (somme des notes des 36 items). Les scores obtenus en ligne de base sur le groupe hôpital de jour (LB groupe HJ) sont comparés aux scores obtenus à la séance 30 pour l'EFC, et sur le groupe après la séance 30 pour l'ECA .

Figure 4

\section{Discussion}

Une nette amélioration est obtenue dès la première séance d'équithérapie, par rapport à la situation de groupe dans le cadre de l'hôpital de jour. Toutes les fonctions impliquées dans le développement sont améliorées, surtout celles concernant la communication, l'imitation, la régulation perceptive, émotionnelle et motrice. La fonction intention est moins nettement améliorée, ce qui amène à penser soit qu'elle est impliquée de façon plus durable dans le dysfonctionnement cognitif, soit qu'elle est moins sollicitée dans cette situation.

Les améliorations sont homogènes pour les six enfants, sans dispersion. L'enfant pour lequel l'amélioration est la moins spectaculaire est celui qui présente un retard associé plus important (retard moyen). Cependant, les bénéfices obtenus pour lui portent sur toutes les fonctions.

Pour les enfants suivis sur 30 séances, les progrès ont perduré, avec une amélioration entre la $19^{\mathrm{e}}$ et la $30^{\mathrm{e}}$ séance. Les bénéfices sont donc progressivement croissants et durables. 
Le poney s'avère être un excellent médiateur, vecteur de tranquillité, de communication, de socialisation. L'enfant se trouve dans une situation lui permettant de mettre en œuvre et de développer ses capacités d'attention, de traitement des informations proprioceptives et extéroceptives, d'association de ces informations, de planification de ses actions, de représentation mentale, afin d'utiliser les moyens adaptés pour atteindre un but, de régulation de ses émotions, de communication ajustée, d'utilisation du langage, d'adaptation au changement.

Il a été surprenant de constater combien ces enfants, d'habitude si déstabilisés par les changements, se sont adaptés sans difficultés aux fréquentes modifications, le plus souvent non prévisibles.

La généralisation des bénéfices en dehors du contexte de l'équithérapie peut être appréciée d'après l'évolution des scores à l'ECA en situation de groupe. L'amélioration est plus nette pour le score «déficience relationnelle », ce qui confirme une généralisation des compétences relationnelles. Évidemment, les autres thérapies proposées à l'enfant interviennent aussi dans ces progrès. Seule une étude sur une grande cohorte avec groupe témoin (enfants recevant les mêmes soins mais sans équithérapie) permettrait d'évaluer le bénéfice propre à ce soin.

L'équithérapie doit s'inclure dans un programme thérapeutique avec un projet personnalisé pour chaque enfant. Elle nécessite un cadre médicalisé, des thérapeutes expérimentés, alliant une formation approfondie dans le domaine des troubles du développement de l'enfant et une très bonne connaissance du cheval, ainsi que l'expérience du travail en partenariat multidisciplinaire. Le thérapeute doit faire preuve de disponibilité et d'adaptabilité, et gérer en souplesse le fonctionnement de la triade enfant/poney/thérapeute.

L'élaboration des objectifs, l'évaluation des résultats et l'analyse des effets bénéfiques permettent de valider la qualité thérapeutique de cette activité et de la valoriser en la différenciant d'une activité de loisir adapté.

\section{Conclusion}

L'activité d'équithérapie permet d'exercer les systèmes d'adaptation de l'enfant à son environnement social et la régulation des fonctions intellectuelles, motrices et émotionnelles. Elle constitue une rééducation efficace des fonctions neuro-psychophysiologiques impliquées dans le développement de la communication et de la socialisation, ainsi que dans la régulation 
cognitive et émotionnelle. Pratiquée dans un climat serein et calme, avec des poneys tranquilles et disponibles, elle permet des échanges adaptés, des acquisitions cognitives, sensorielles et motrices, et constitue une situation «anti-autistique »: l'enfant est stable, apaisé par les mouvements du poney, il sollicite l'équithérapeute dans une interaction adaptée et prend conscience de l'effet de ses actions sur le poney, excellent cothérapeute favorisant aussi l'attachement.

Cette thérapie, en dehors de l'hôpital mais en lien étroit avec les soins réalisés au sein de l'hôpital, apporte aussi des bénéfices plus larges pour l'enfant (épanouissement, plaisir, affirmation de soi, autonomie), et pour les parents (ouverture de leur enfant à une activité ordinaire).

Ces résultats positifs nous ont amené à élargir les indications à des enfants présentant des troubles de l'adaptation sociale, des troubles hyperkinétiques, des troubles émotionnels, associés ou non à des conditions psychosociales défavorables.

\section{Conflit d'intérêt : à compléter par l'auteur}

\section{Références}

[1] Adrien JL, Roux S, Couturier G, Malvy J, Guerin P, Debuly S, et al. Towards a new functional assessment of autistic dysfunction in children with developmental disorder. The behaviour Function Inventory. Autism 2001;5:249-64.

[2] Barthelemy C, Hameury L, Lelord G. L'autisme de l'enfant. La thérapie d'échange et de développement. Paris: Expansion Scientifique Française; 1995.

[3] Barthelemy C, Roux S, Adrien JL, Hameury L, Guerin P, Garreau B, et al. Validation of the Revised Behavior Summarized Evaluation Scale. Journal of Autism and developmental disorders 1997;27:139-53.

[4] Beiger F. L'enfant et la médiation animale. Une nouvelle approche par la zoothérapie. Paris: Dunod; 2008.

[5] De Lubersac R, Lallery H. La rééducation par l'équitation. Paris: Crepin-Leblond; 1973.

[6] De Lubersac R, et al. Thérapies avec le cheval. Paris: Fentac; 2000.

[7] Faucon CE. Équitation thérapeutique et pédopsychiatrie (thèse). Paris: Université Paris VI; 1977.

[8] Hameury L, Lenoir P, Bodier C, Sauvage D. Les thérapies des troubles du développement du jeune enfant. Rev Med Tours 2000;34:137-9. 
[9] Hameury L, Bougard PM. Bilan de l'activité d'équitation thérapeutique. Handicheval magazine, Bulletin d'information de la Fédération Nationale Handi Cheval 2005;3-4:4-11.

[10] Jollinier M, et al. Cheval, Inadaptations et Handicaps. Paris: Maloine; 1995.

[11] Schopler E, Reichler R, Renner BR. The Childhood Autism Rating Scale (CARS) for Diagnostic Screening and Classification of Autism. New York: Irvington; 1986.

Figure 1

EVALUATION EFC - score global et par enfant 6 enfants - 19 séances

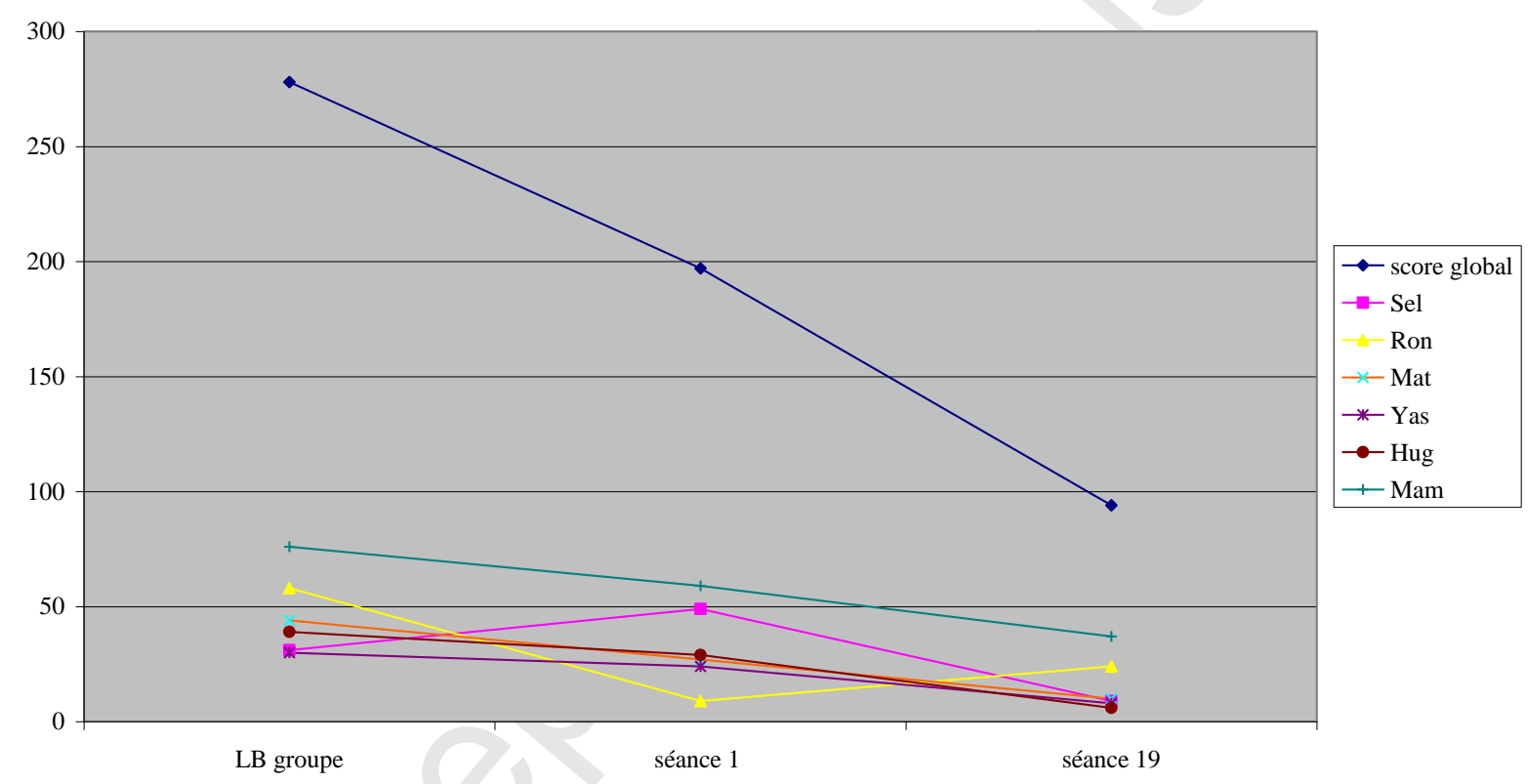

Figure 2 


\section{EVALUATION EFC - score par fonction \\ 6 enfants - 19 séances}

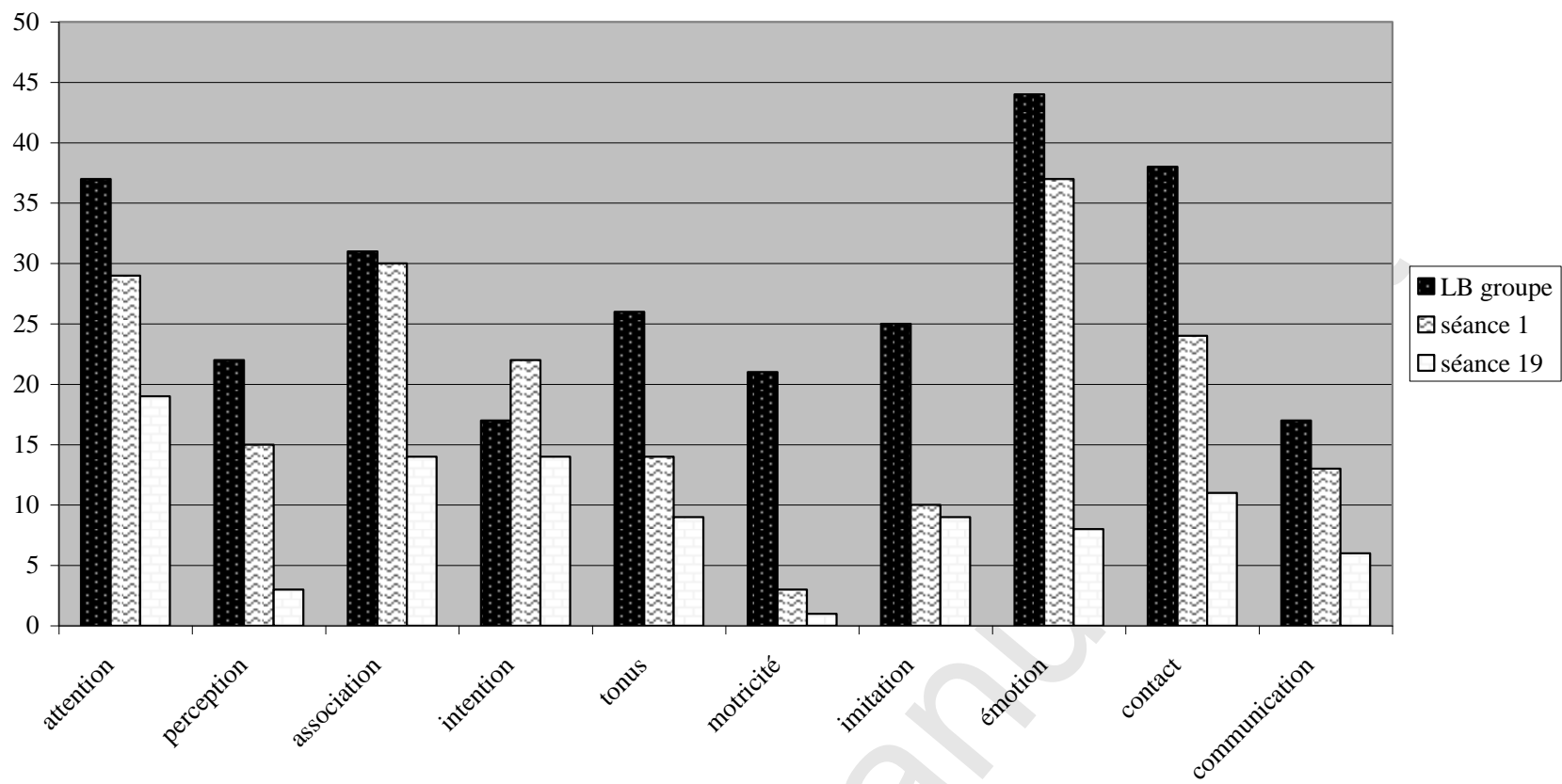

Figure 3

EVALUATION ECA groupe - score déficience relationnelle global et par enfant 6 enfants - 19 séances

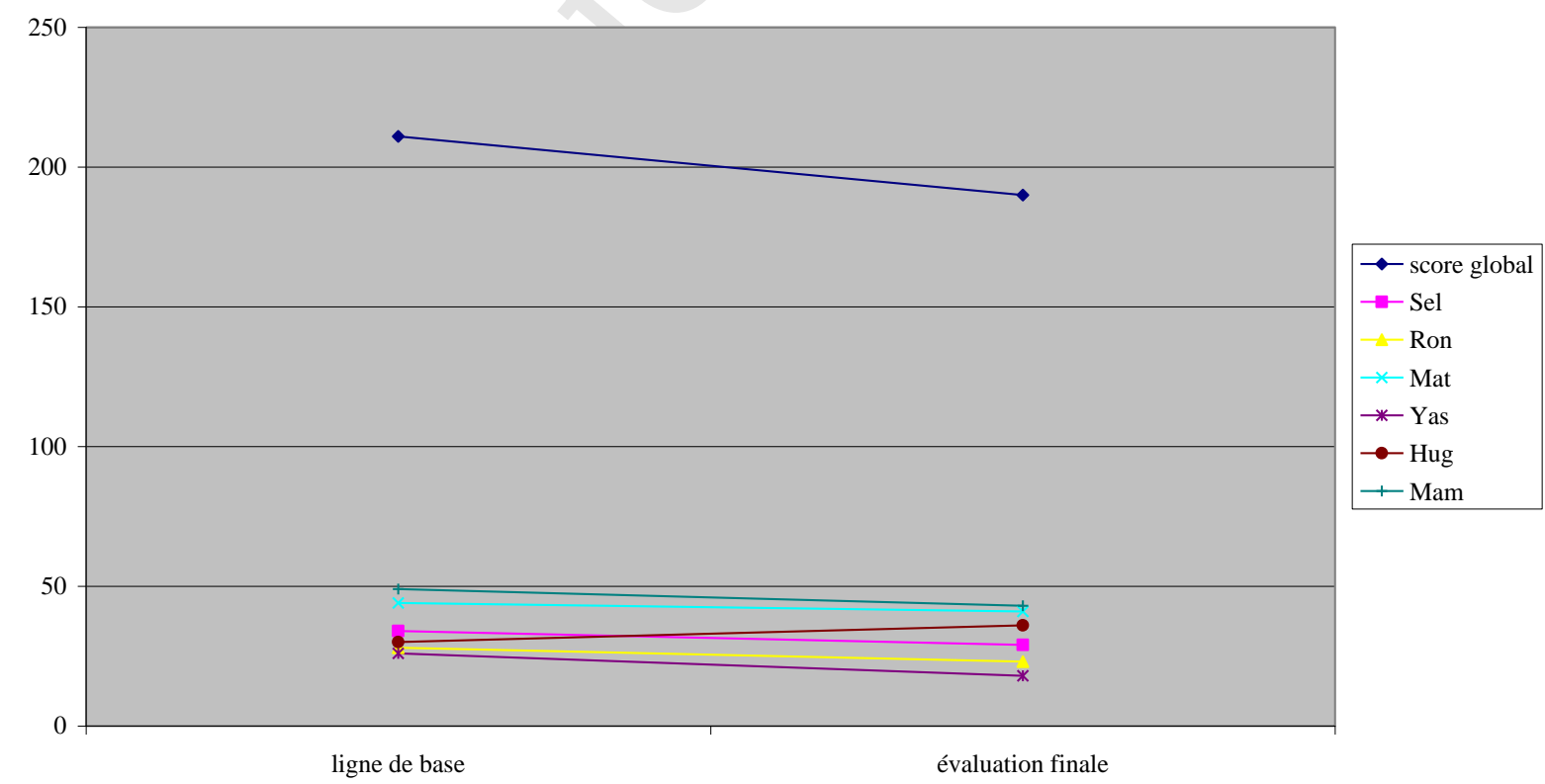

Figure 4 
4 enfants - 30 séances

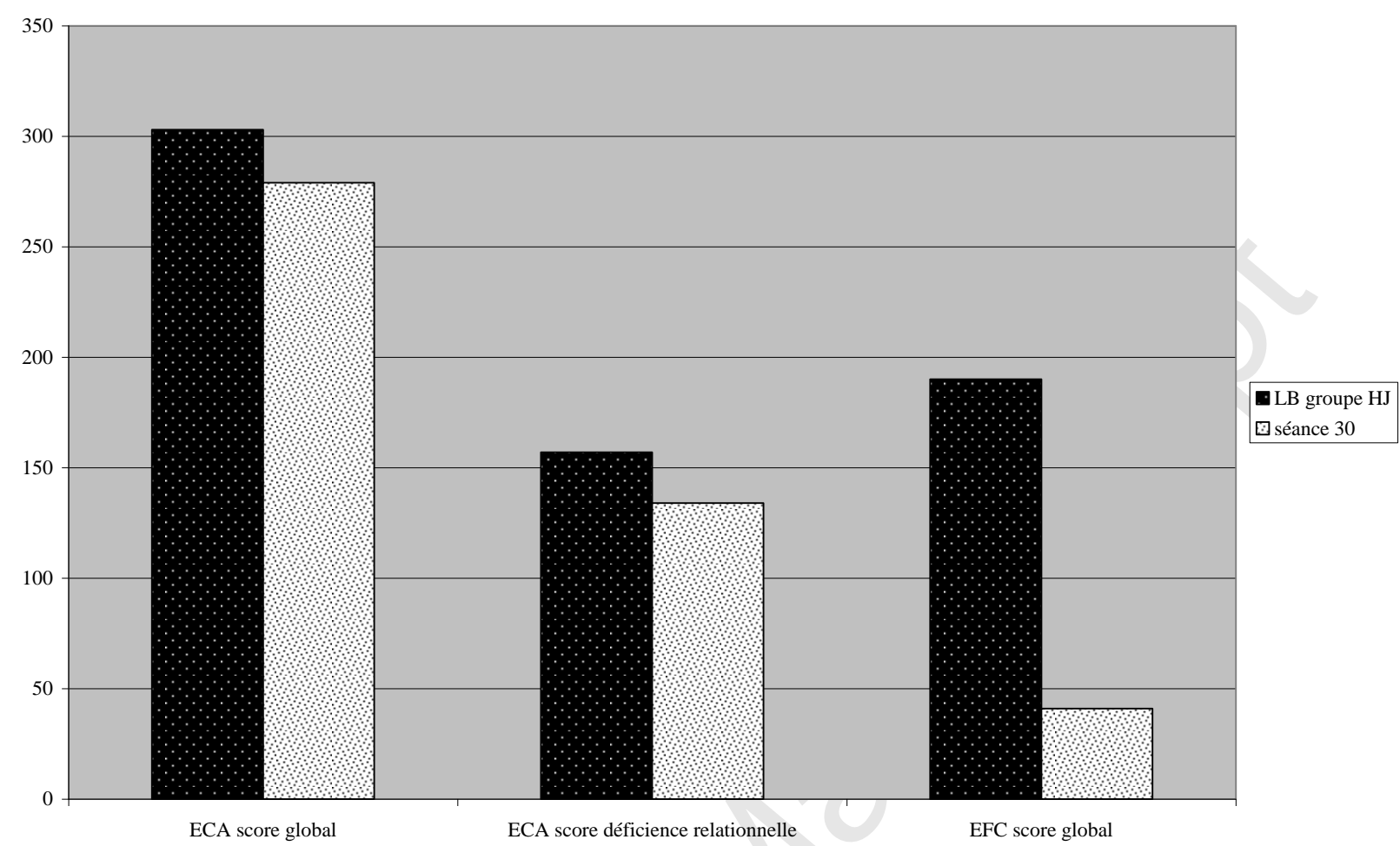

